\title{
PREVAlLing wind blows more good news for enzalutamide
}

Analysis of data collected during the PREVAIL trial has indicated a significant improvement in healthrelated quality of life (HRQOL) and a delay in skeletal-related events in men treated with enzalutamide for metastatic castration-resistant prostate cancer (mCRPC). The data were published in The Lancet Oncology.

\section{4 ...these new data can only bolster enzalutamide's potential and reputation... 77}

The PREVAIL trial is a phase III, randomized, double-blind trial, in which chemotherapy-naive patients with prostate cancer that had progressed despite androgen deprivation received either oral enzalutamide $160 \mathrm{mg} /$ day $(n=872)$ or placebo $(n=845)$. Previously published data from PREVAIL showed a substantial improvement in overall survival in patients who received enzalutamide versus those on placebo, and a delayed need for chemotherapy. In this analysis, HRQOL was assessed using the Functional Assessment of Cancer Therapy-Prostate (FACT-P) and EQ-5D questionnaires, at baseline and during treatment, and pain was assessed using the Brief Pain Inventory Short Form questionnaire at screening, baseline, week 13 and week 25 of the study. Using these data, the researchers investigated the change from baseline in these parameters, percentage improvement and time to deterioration in pain and HRQOL, as well as the proportion of patients experiencing a skeletal-related event and the time to first skeletal-related event (defined as radiotherapy or surgery to the bone for prostate cancer, pathological fracture, spinal cord compression, or change in antineoplastic therapy to treat bone pain).

Median duration of therapy was longer in the enzalutamide group than in the placebo group (16.6 months versus 4.6 months), reflecting the increased efficacy of enzalutamide over placebo, as has been demonstrated in previous data produced from the PREVAIL trial, which showed a substantial survival benefit and resulted in the trial being halted and unblinded early, in order to enable men who had been randomized to placebo to receive enzalutamide therapy. Evaluation of HRQOL using FACT-P and EQ-5D showed significant differences in HRQOL change from baseline to week 61 in patients receiving enzalutamide compared with those receiving placebo, with a median time to deterioration in FACT-P score of 11.3 months in the enzalutamide group and 5.6 months in the placebo group $(P<0.0001)$. By the end of data collection, 278 of patients in

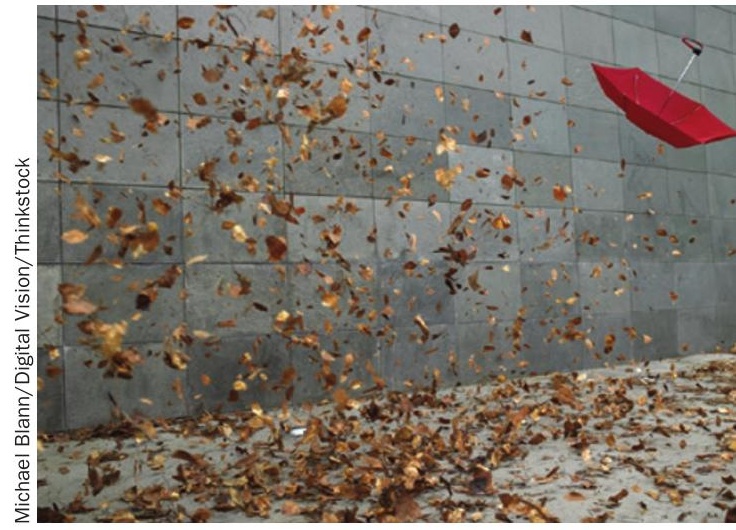

the enzalutamide group (32\%) and 309 of 845 men in the placebo group (37\%) had experienced a skeletal-related event, with a longer time to first skeletal-related event in enzalutamide-treated men.

Building on the previously published data from PREVAIL, these new data can only bolster enzalutamide's potential and reputation as an excellent therapeutic option for men with mCRPC. According to the study authors, further analyses to define the relationship between objective clinical responses, surrogate markers of efficacy, and patient-reported outcomes are ongoing.

Annette Fenner 